\section{$(3)$ HORIZONTES}

www.revistahorizontes.org
Horizontes. Revista de Investigación en Ciencias de la Educación Https://doi.org/10.33996/revistahorizontes.v4i15.112 Julio - Septiembre 2020 Volumen 4 / No. 15 ISSN: $2616-7964$ ISSN-L: $2616-7964$ pp. $246-257$

\title{
La corresponsabilidad social de los medios radiales en la educación virtual en tiempos de pandemia
}

The social co-responsibility of radio media in virtual education in times of pandemic

A corresponsabilidade social da mídia do rádio na educação virtual nos tempos da Pandemia

ARTICULO ORIGINAL

Verónica Medina Ayala

maribelmedinaayala@gmail.com

ORCID: 0000-0003-3062-3447

\author{
Verónica Peñaherrera Garzón \\ veroka2003@hotmail.com
}

ORCID: 0000-0003-0634-9337

Instituto Superior Tecnológico “Vicente León”, Ecuador

Recibido abril 2020 | Revisado mayo 2020 | Publicado en julio 2020

\section{RESUMEN}

A nivel mundial, la educación cambió sustancialmente a raíz de la pandemia COVID-19, obligando a estudiantes y maestros a depender de la tecnología para impartir clases y continuar con el proceso enseñanza y aprendizaje, sin embargo, no todos tienen el privilegio de acceder a la educación virtual. El objetivo principal es analizar la corresponsabilidad social de los medios radiales de la ciudad de Latacunga en la educación virtual en tiempos de pandemia, el propósito es llegar a lugares donde el internet es limitado. La muestra poblacional fueron gerentes y periodistas de los medios radiales de la localidad. Ecuador como parte de la investigación descriptiva usando como técnica el análisis cualitativo y cuantitativo. Los resultados obtenidos en las encuestas fueron la poca responsabilidad social y corresponsabilidad de los medios de comunicación radiales, siendo que, su rol principal es educar, informar y entretener, según la Ley de Comunicación vigente en el país.

Palabras clave: Corresponsabilidad social; medios radiales; educación virtual; pandemia; Covid-19

\section{ABSTRACT}

Globally, education changed substantially as a result of the COVID-19 pandemic, forcing students and teachers to depend on technology to teach classes and continue the teachinglearning process, however, not everyone has the privilege of accessing virtual education. The main objective is to analyze the social co-responsibility of the radio media of Latacunga city in virtual education in times of pandemic, the purpose is to reach places where the internet is limited. For this reason, managers and journalists from the radio media of the city of Latacunga were considered as a population sample, as part of the descriptive research with a qualitative quantitative approach. The results obtained in the surveys were the little social responsibility and co-responsibility of the radio communication media in the aforementioned issue, being that their main role is to educate, inform and entertain, according to the Communication Law in force in the country.

Key words: Social co-responsibility; radio media; virtual education; pandemic, Covid-19 


\section{RESUMO}

No mundo todo, a educação mudou substancialmente a raiz da pandemia Covid-19, fazendo que os estudantes e os mestres tenham que depender da tecnologia para transmitir classes e seguir com os processos de ensinoaprendizagem, porém, nem todos podem ter o privilégio de acessar à educação virtual. 0 objetivo principal é analisar a corresponsabilidade social da mídia das rádios da cidade de Latacunga na educação virtual em tempos de pandemia, o objetivo é chegar nos lugares onde a internet é limitada. Assim sendo, foi considerado como amostra da população os gerentes e jornalistas da mídia radial da cidade de Latacunga, como parte da investigação descritiva focada nos aspectos quali-quantitativos. Os resultados obtidos nas enquetes foram a pouca responsabilidade e corresponsabilidade da mídia da radio, no tema referido, sendo que, sua função principal é educar, informar e entreter, segundo a Lei de Comunicação válida no país.

Palavras-chave: Corresponsabilidade social; mídia da radio; educação virtual, pandemia Covid19

\section{INTRODUCCIÓN}

La declaración mundial de una pandemia transformó abruptamente las condiciones de la educación, cambió inmediatamente de la modalidad presencial a la virtual, causando complicaciones, dilemas y dificultades como: la despersonalización del mensaje en las clases, el doble trabajo para los docentes; pues por un lado deben preparar la cátedra a través de videos interactivos y por otro, realizar módulos del contenido de la materia para quienes no tienen acceso al internet $y$ finalmente la dificultad del alumno de afianzar la teoría con la práctica al no tener una respuesta inmediata del profesor como ocurría en la educación presencial.

En Ecuador, el acceso a las clases virtuales es limitado, pues, en varios lugares, su cobertura es deficiente, la señal no llega en su totalidad especialmente en el área rural tanto de la costa como de la sierra, al estar ubicados a distancias lejanas de la ciudad y a su vez, están rodeados de montañas lo que impide una buena conexión, de esta manera, la eficacia del proceso enseñanza $y$ aprendizaje ha disminuido considerablemente.

Dentro de este marco, la educación necesita de aliados estratégicos para poder cumplir su misión, es por esto que, los medios de comunicación social especialmente los radiales, serán quienes asuman el reto de no solamente informar y entretener, sino el más importante, el de educar, a través de programas puntuales que refuercen lo aprendido en las clases virtuales con el propósito de que predomine un trabajo de orientación y ofrecerle mecanismos que le permitan ir explorando la materia.

Para esto, es importante que exista una relación de cercanía entre la persona que locuta un programa y quien lo escucha, para Rudolf Arnheim en su libro Estética Radiofónica, precisa que:

La radio es el medio que más se acerca a nuestra intimidad, en tanto habla al oído, se dirige a uno de los órganos más sensibles, pero las relaciones de cercanía no se logran sólo por ese hecho, se cultivan también a través de los recursos: la interlocución, la palabra y las rutinas (Arnheinm, 1979, s/p).

Lograr relaciones de cercanía no significa solo hablar de los problemas de la gente, significa hacerlo mediante recursos discursivos de acercamiento. En la actualidad, los avances tecnológicos permiten que los medios de comunicación tengan mayor cobertura, es por esto que las frecuencias: amplitud modulada o frecuencia modulada se han transformado y son sintonizadas a través de las radios online, sin embargo, no solo la radio ha tenido este 
impulso, según Figueroa (1996) "las políticas gubernamentales, las acciones de los concesionarios son otros elementos que intervienen en su transformación". (Arteaga, 2004). Es importante que exista este estímulo tanto con la radio, estado y familia ya que, ayudarán a mejorar la comprensión de la información que se emite en los programas radiales.

Según la Ley Orgánica de comunicación (LOC) en el artículo 71 menciona que:

La información es un derecho constitucional y un bien público; y la comunicación social que se realiza a través de los medios de comunicación es un servicio público que deberá ser prestado con responsabilidad y calidad, respetando los derechos de la comunicación establecidos en la Constitución, los instrumentos internacionales y contribuyendo al buen vivir de las personas. (LOC, 2013).

Es importante hacer referencia al rol que tienen los medios de comunicación, pues al ser un servicio público están obligados a satisfacer ciertas necesidades de la población y entre ellas está la de informarse, entretenerse y educarse.

De igual manera, en el artículo 71, inciso 10 especifica que se debe propender a la educomunicación, sin embargo, en Ecuador y específicamente en el cantón Latacunga , no se ha palpado que exista esta responsabilidad por parte de los medios en la programación de las emisoras, dando paso a que esta política pública sea muy "tibia" y si a esto se agrega que se necesita de recursos económicos para poder realizar las producciones, es más complicado aún que se cumpla a cabalidad con la tan anhelada educación a través de la radio, es por esto que la corresponsabilidad debe ser también con el estado, siendo este, el ente principal para destinar los fondos necesarios para que pueda cumplirse con lo que exige la mencionada ley.

Trabajar por la educación es una tarea difícil y más aún en la modalidad virtual, pues esa interrelación docente y estudiante es fundamental, ya que, existe el intercambio de conocimientos y el intercambio de saberes entre estos dos actores, sin embargo, por algunos factores, este proceso no se cumple al cien por ciento.

Para que cumpla con lo anteriormente dicho, según (Hurtado, 2020) recomienda para que la educación del siglo XXI se convierta en un motor de desarrollo, debe:

- Ser flexible y lo más alejada posible de modelos rígidos, pues el mundo cambia constantemente $\mathrm{y}$ es preciso que se adapten a nuevos contextos $\mathrm{y}$ circunstancias.

- Es necesario que se promueva valores sociales como la igualdad la justicia, la cooperación y la ayuda humanitaria, pues de esta forma se anima a los ciudadanos del mañana a ser motores de cambios estructurales y a tomar conciencia de las necesidades reales de su entorno.

- Debe insistir en el modelo de desarrollo sostenible como una meta a la que todos debemos contribuir

Es necesario mencionar que el tema de la educomunicación no es un tema nuevo, pues entre los años 50 a 70, desarrollaron el trabajo de educación para adultos con las llamadas escuelas radiofónicas, siendo el Instituto Radiofónico Fe y Alegría (IRFEYAL) de la ciudad de Quito, "los brazos ejecutores de las políticas educativas del Ministerio de Educación especialmente de la educación a distancia y presencial para adultos" (IRFEYAL, 2014) y fue así que esta emisora educativa formó a cientos de alumnos adultos en varias profesiones, 
lastimosamente hoy por hoy, esta modalidad educativa ha perdido su fuerza.

Ahora bien, la educación ha tomado otro camino y se ha visto obligada a ajustarse a un nuevo escenario formativo, demanda cambiar la percepción de educación tradicional, en donde el maestro dictaba su clase únicamente apoyándose en la pizarra como herramienta didáctica, ahora, la convergencia digital es abismal, el docente debe transformarse y ser creativo; de lo contrario los resultados que obtenga con sus alumnos, no serán los más idóneos, además, se depende al cien por ciento de la conectividad, si ésta falla, las estrategias para generar un proceso de enseñanza -a aprendizaje idóneo, no servirán de nada.

A diario, los docentes del Instituto Superior Tecnológico "Vicente León" enfrentan este tipo de problemas, por una parte, la cobertura no es adecuada o definitivamente no tienen acceso a la conexión WIFI en su hogar, tendiendo que utilizar los datos móviles del celular y, por otro lado, estudiantes que no tienen acceso a dispositivos electrónicos para asistir a clases, sin mencionar que los que tienen, deben pagar datos móviles o acudir a otras instancias para utilizar la señal del internet, lo que provoca desmotivación y preocupación al no tener las garantías suficientes de conectividad.

Por esta razón, el objetivo principal de la investigación es analizar la corresponsabilidad social de los medios radiales de la ciudad de Latacunga en la educación virtual en tiempos de pandemia, a través de un estudio individual de la plataforma de programación de los medios de comunicación, pues, por su nivel de alcance, el propósito es llegar a lugares donde el internet es limitado, dando así más oportunidad a que las personas en su mayoría accedan a la educación.

Es indispensable buscar alternativas para que esta brecha sea cada día menor, una de ellas es crear un espacio en un medio radial de mayor sintonía de la localidad y así, poder transmitir clases interactivas sobre las cátedras establecidas dentro de la malla curricular de las 6 carreras que son: Tecnología Superior en Marketing, Administración Financiera, Seguridad e Higiene del Trabajo, Contabilidad y Gastronomía con que cuenta el Instituto Superior Tecnológico "Vicente León" de la ciudad de Latacunga.

Por esta razón, la importancia de este estudio se fundamenta en el proceso de enseñanza- aprendizaje, involucrando a los responsables de los medios de comunicación radiales, comunidad educativa y sus familias, pues, lamentablemente, con la modalidad actual de educación virtual, el maestro por sí solo, no podrá conseguir este propósito.

Para Villoro (1989) en su libro Creer, saber, conocer enfatiza que cuando enseñamos, esperamos que nuestros alumnos adquieran un conocimiento; pero eso no siempre es posible, porque requerirían de la experiencia directa y en muchos casos, solo pueden adquirir un saber.

Razón por la que, el docente tiene la responsabilidad de tratar dos aspectos en cada una de sus clases, el saber y el conocer pues la enseñanza que se imparte debe tener la teoría y la práctica, considerando canales alternativos a la educación en línea y las limitaciones comunicacionales que se evidencia a diario.

\section{MÉTODO}

Para este estudio se consideró a la investigación descriptiva, seleccionando las características fundamentales del objeto de estudio, para Cesar Bernal en su libro Metodología de la Investigación, menciona que "se deben describir aquellos aspectos más característicos, distintivos y particulares de estas personas, situaciones o cosas, o seas, aquellas propiedades que las hacen reconocibles a los ojos de los demás". (Bernal, 
2006, p. 117), además, se analizaron los datos bajo la técnica cualitativa y cuantitativo ya que se realizaron 2 entrevistas semiestructuradas a los gerentes de los medios radiales que fueron seleccionados por su dominio en el ámbito radial y 19 encuestas a periodistas y comunicadores sociales de la localidad, siendo ellos, la muestra aleatoria de las 7 radios existentes en la ciudad de Latacunga.

El estudio se llevó a cabo con una población total de 7 radios de la ciudad de Latacunga, entre las que se encuentran: Radio Latacunga, Elite, Municipal, Cotopaxi, Novedades, Nuevos Horizontes, Hechizo y 19 profesionales en Comunicación Social o Periodismo que trabajan en mencionados medios de comunicación radiofónicos.

\section{RESULTADOS}

La importancia de la radio como herramienta en tiempos de PANDEMIA trata de los resultados arrojados de la aplicación y el análisis más significativo de las encuestas a los periodistas y comunicadores sociales del cantón
Latacunga. Al mismo tiempo se muestran las impresiones recogidas mediante la entrevista semiestructura a los gerentes de los medios radiales que fueron seleccionados.

En donde se destacó que la radio como herramienta educativa, según resultados señalados manifestados en el estudio en relación a la totalidad de los comunicadores sociales encuestados consideraron que es una herramienta estratégica en tiempos de pandemia, ubicando a la radio como un medio alternativo a la educación virtual dirigido a alumnos que no tienen accesibilidad a este servicio.

Posteriormente, en concordancia a la radio como un recurso para la práctica docente, (Figura 1) los resultados obtenidos, enfatizaron que el $84,2 \%$ de encuestados manifestaron que si están de acuerdo, mientras que el otro 15,8\% determinó que no es un recurso adecuado, estableciendo la importancia de inmiscuir la radio en la práctica docente en los lugares donde la conectividad no existe.

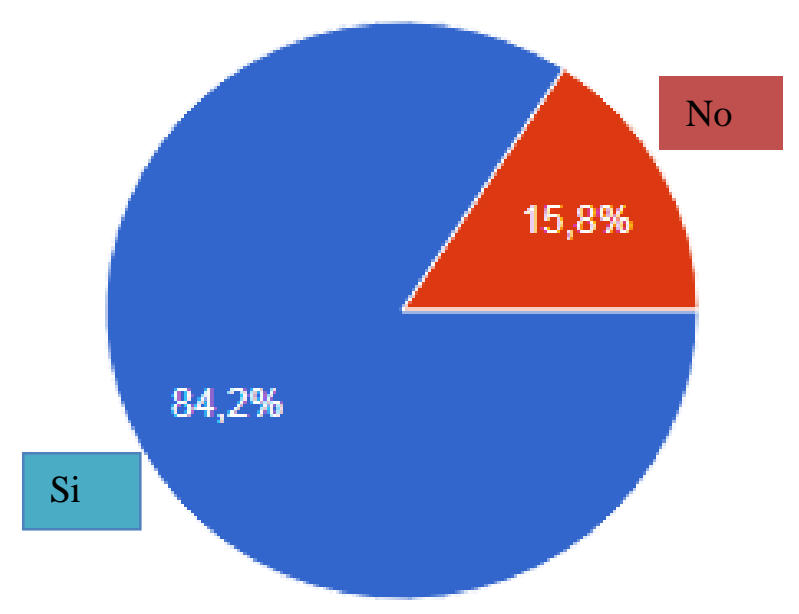

Figura 1. La radio como un recurso para la práctica docente. 
Como puede observarse en la figura 2 en correspondencia en que si la enseñanza a través de la radio reemplaza a la educación virtual en tiempo de Pandemia, el 57,9 \% consideraron que si es una alternativa válida, mientras que, el $42.1 \%$ establece que es insustituible, tomando los resultados expuestos, es de vital importancia generar procesos de enseñanza a través de la radio, teniendo en cuenta que es un medio de comunicación con gran alcance.

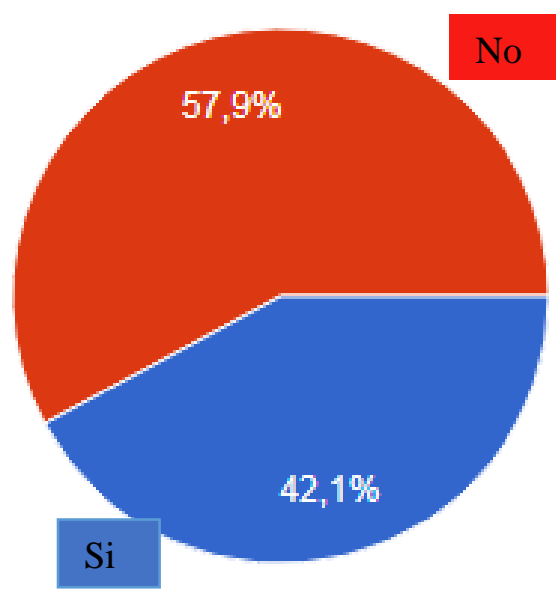

Figura 2. La enseñanza a través de la radio como una alternativa a la educación virtual.

A continuación se establece el aporte de los medios de comunicación radiales en la ciudad de Latacunga en educación virtual, (Figura 3) según los datos que arrojó la encuesta los programas de educación gratuitos representa el $42 \%$, cuñas educativas el $26,3 \%$, campañas informativas con el 15 , $8 \%$ y el otro $15,8 \%$ no

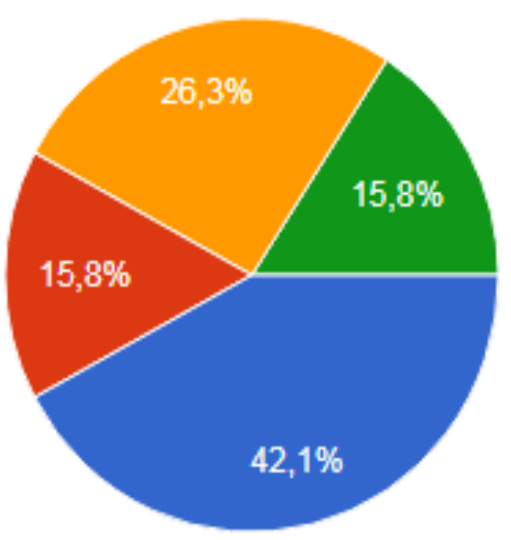

cuenta con ningún aporte en el área educativa. Estos resultados manifiestan que las frecuencias son de propiedad del Estado, entregado a través de la concesiones a figuras administradas, por ende se debe incentivar a generar programas educativos de interés colectivo.

Figura 3. Corresponsabilidad de los medos de comunicación radiales en el proceso de educación virtual.

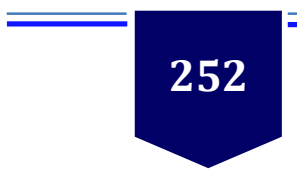


En cuanto a la cantidad y el tiempo de la programación educativa establecida en medios de comunicación radial, el 57,9\%, declararon que un programa, luego con un $15,8 \%$ dos programas, seguido de un 5,2 \% tres programas educativos, mientras que el 15,8 no cuenta con aportes a la educación en su programación,
(Figura 4) con los resultados equitativos es preciso recordar a los gerentes o propietarios de medios de comunicación radiales la designación de una hora diaria, no acumulable para programadas de educación, establecido en la Ley de Comunicación en Ecuador.

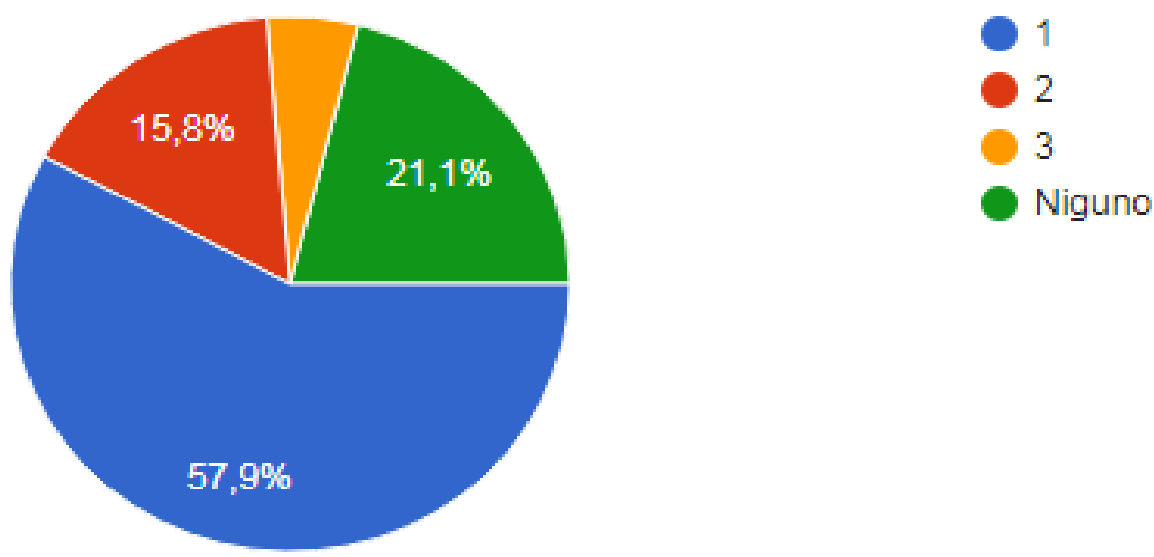

Figura 5. Programación educativa en medio de comunicaciones radiales en tiempo de Pandemia.

Para finalizar con el análisis mostrado a lo largo del desarrollo del estudio que se llevó a cabo, se tiene que la corresponsabilidad social en educación se cumple en el 50\%, mientras que el otro $50 \%$ restante no cumple con la política pública, (Figura 5) según los datos que

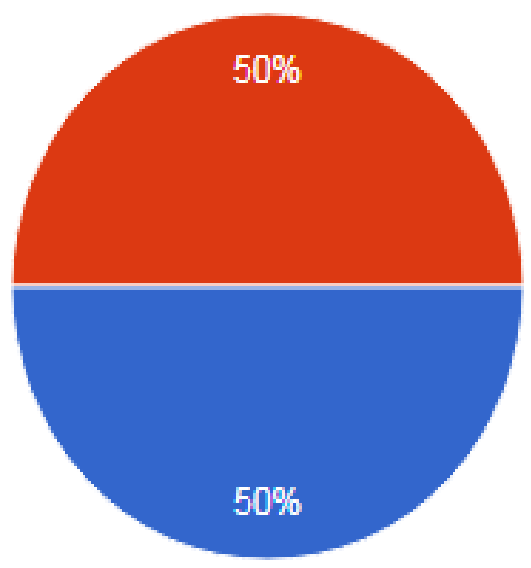

arrojó el estudio debido a la realidad que vive la educación en tiempos de crisis, los docentes tienen la responsabilidad de buscar estrategias y canales alternativos como la radio para llegar con el conocimientos a todos los estudiantes en igualdad de condiciones.

Figura 5. Programas gratuitos de corresponsabilidad en educación en radios de Latacunga. 
En una entrevista realizada a Eduardo Guerrero, (2020), profesional en el área de la Comunicación, este recalcó la importancia de los medios de Comunicación Social, en especial radiofónicos como una herramienta alternativa para la educación en tiempo de crisis, teniendo como antecedente que la radio es un medio de servicio al Estado (ciudadanía), pues, la Agencia de Regulación y Control de las Telecomunicaciones entrega una frecuencia para su administración en beneficio de la ciudadanía ecuatoriana, más no, para el servicio de sus gerentes o administradores.

En los últimos años se ha podido evidenciar que los medios de comunicación social son consideradas empresas generadoras de lucro, convirtiendo al espectro radioeléctrico en empresas comerciales, a pesar de ser considerado un sector estratégico, que el Gobierno se reserva el derecho de su administración, regulación, control y gestión.

Recordó que las políticas púbicas establecidas en la Ley de comunicación en el Art. 74 literal d, establece Destinar una hora diaria, no acumulable para programas oficiales de teleeducación, cultura, salubridad y derechos elaborados por los Ministerios o Secretarías con competencia en estas materias.

No existe el cumplimiento de esta disposición, por lo que, la mayoría de medios de comunicación radiales de la provincia de Cotopaxi y del país, tienen como prioridad la entrega de información, y como actores o protagonistas de esto a los políticos, personas e instituciones que pagan por la trasmisión de información, monopolizado la información, dejando a un lado la razón de ser de la radio y su responsabilidad social en el tema educativo (Tabla 1).

Tabla 1. Trilogía de la educación en tiempos de crisis a través de la radio

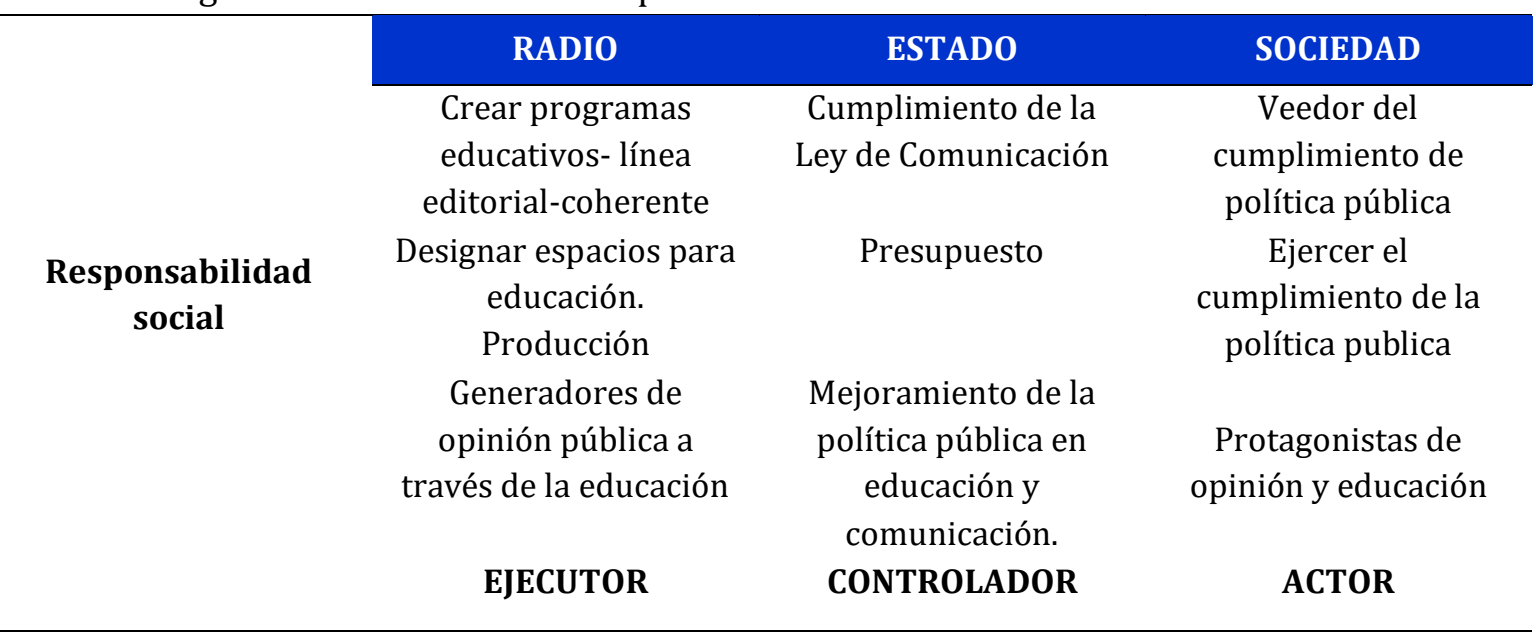

La importancia de considerar la radio como una herramienta de educación alternativa a la educación virtual, a través del cumplimiento de la política pública establecida por el Gobierno Nacional en la Ley de Educación y su relación con los administradores de las frecuencias radiofónicas y la sociedad como actores fundamentales de la educación y formación de hombre y mujeres generadores de opinión.

Guerrero (2020) insistió en el trabajo ejecutado en los años 70 a través de las Escuelas Radiofónicas Populares (ERPE), consideradas como una extensión del aula para educar a través de la radio en lugares donde el acceso fisco era imposible, mientras 
que la radio rompe barreras de distancia geográficas y accesibilidad, cuyo objetivo primordial es el servicio educativo, social y de desarrollo, cuya opción es el compromiso con los sectores populares del campo y de la ciudad, de modo particular con el sector menos favorecidos.

A pesar de las nuevas tecnologías y las redes sociales que han mediatizado la información y la comunicación, la radio sigue siendo una parte fundamental en la vida de los ecuatorianos, por ello es primordial retomar el verdadero sentido de la Radio dirigido a la educación en concordancia con la familia y el estado. Para finalizar indicó que durante la emergencia sanitaria ningún medio de comunicación local se ha preocupado por generar contenido educativo que contribuya con la crisis de salud, más bien, se ha priorizado la información unidireccional de acontecimientos y hechos noticiosos dejando a un lado la responsabilidad social con la educación y el reto enfrenta docentes y estudiantes

Sonia Guaigua (2020), profesional en el área de comunicación, en la entrevista ejecutada para esta investigación expuso la importancia del papel de los profesionales del área de la Comunicación en el cumplimiento de las políticas públicas establecidas en la Carta Magna y en la Ley de Comunicación, siendo los responsables de generar productos comunicacionales enfocados a una de las razones de ser de la profesión y de todos los medios de comunicación, como es educar.

Guaigua (ob. cit) recordó que desde el año 1950 el Instituto Radiofónico Fe y Alegría (IRFEYAL, 2014), aposto a la educación a través de la Radio, asumiendo un trabajo para combatir el analfabetismo en las personas adultas, de esta manera IRFEYAL se convierte en uno de los brazos ejecutores de las políticas educativas del Ministerio de
Educación, en especial de la educación a distancia para adultos.

Desde 1986, IRFEYAL emite clases radiofónicas a sus alumnos, a través de una onda media con una potencia de $5 \mathrm{kw}$, además contaba con instalaciones necesarias para la producción de clases a través de Radio IRFEYAL "La Voz de Fe y Alegría" 1090 Amplitud Modulada en la ciudad de Guayaquil, este es un gran ejemplo de organización entre el estado, empresas radiofónicas, ciudadanía y profesionales para contribuir con la educación, finalizó.

\section{Discusión}

Es necesario partir de la importancia de los medios como escuelas paralelas, según Aguaded (1995) se centra en la influencia de los medios de comunicación en la formación del pensamiento de los niños y en la socialización del sujeto a lo largo de su vida, alcance aspectos como la identidad cultural, el consumismo, la difusión de modelos extranjerizantes, la manipulación ideológica de la información, entre otros.

Durante los dos últimos períodos académicos del año 2020, estudiantes y docentes han enfrentado un gran reto como causa de la emergencia sanitaria por el COVID-19, cambiando las clases presenciales por virtuales, dependiendo en un $100 \%$ de la tecnología- o conectividad, como herramienta primordial para el desarrollo de las mismas. A pesar de que muchas familias no cuentan con acceso a este servicio, es ahí, donde la educación y el Gobierno Nacional enfrentan un reto muy grande.

Según Guerrero (2020) en la búsquedas de nuevas estrategias que permitan que la educación llegue a todos los estudiantes, independientemente de su acceso a la tecnología, la radio se presenta como una herramienta alternativa en la construcción de 
una sociedad y el impacto que genera los mensaje que se difunden, sin embargo, la generación de productos comunicacionales educativos no han incrementado a pesar de contar con una Ley de Comunicación, que claramente establece en su Art. 74 literal (d), la obligación de todos los medios de comunicación a destinar una hora diaria, no acumulable para programas oficiales de tele-educación, cultura, salubridad entre otros.

Ante el no cumplimiento de esta política pública se busca acrecentar la corresponsabilidad de los medios de comunicación especialmente radiales, pues, por su nivel de alcance, permitirá llegar a lugares donde el internet es limitado, dando la oportunidad a que muchas personas puedan acceder a la educación.

Trabajar en la educación virtual es una tarea difícil, y más aún cuando no se cuenta con las herramientas necesarias para generar el intercambio-realimentación de conocimientos, sin embargo, por algunos factores, este proceso no se cumple al cien por ciento a nivel presencial y menos a nivel virtual, por ello, es importante la búsqueda de tácticas que se asemejen a una educación presencial efectiva en tiempos de crisis.

Al respecto de esta necesidad latente, Instituto Radiofónico Fe y Alegría (IRFEYAL) emprendió sus actividades educativas atendiendo a los sectores menos favorecidos, de
Quito, Imbabura, Carchi y Loja donde desarrollo los primeros programas radiofónicos de alfabetización, en el año de 1977, con la participación de 1025 alumnos, de ellos el 76\% mujeres y el 23\% hombres, con la cooperación de 4 emisoras colaboradoras. (IRFEYAL, 2014).

Por ello, es indispensable retornar estrategias empleadas por fundaciones privadas donde su principal herramienta de trabajo para educar se basaba en la radio. En esta crisis sanitaria en especial en los lugares donde la conectividad no es permanente la radio se convierte en un aleado estratégico para poder cumplir con el proceso educativo, es por esto que, los medios de comunicación social especialmente los radiales, serán quienes asuman el reto de no solamente informar y entretener, sino el más importante, el de educar.

En general, cuando desde una planificación educativa se piensa en la necesidad de incorporar medios de comunicación como herramientas para crear materiales educativos y medios de transmisión de dichos contenidos se crea una sinergia entre la educación y la comunicación.

Identificar los actores y requerimientos para una educación alternativa en tiempos de crisis, es fundamental, la asignación de presupuesto por parte del Gobierno Nacional y las empresas radiofónicas es fundamental para garantizar el acceso equitativo a la educación en tiempo de crisis. (Figura 6). 


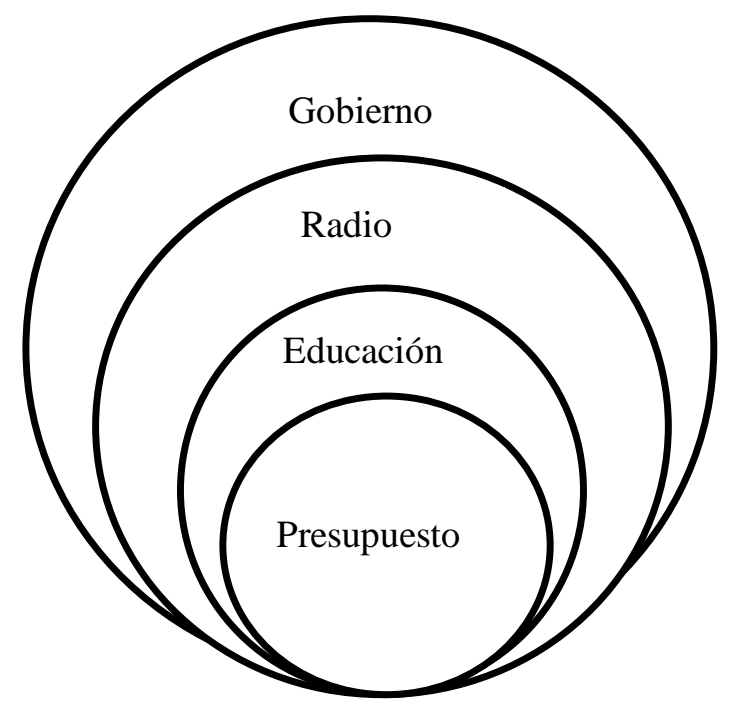

Figura 6. Actores y requerimiento para una educación alternativa en tiempos de crisis

\section{CONCLUSIONES}

Una vez analizados los resultados obtenido se determinó el cumplimiento del objetivo de la investigación e cual fue analizar la corresponsabilidad social de los medios radiales de la ciudad de Latacunga en la educación virtual en tiempo de pandemia, en donde se evidenció falencias en el cumplimiento de las políticas públicas por parte de los gerentes de las radios y el incumplimiento de la ley de Comunicación, así como la falta de seguimiento por parte del Gobierno Nacional, en la designación de espacios radiales no acumulativos a favor de la educación. En consecuencia, se demuestra la falta de corresponsabilidad social de quienes hacen radio en la ciudad de Latacunga, Ecuador a favor de la educación, a pesar de considerar que la radio es una herramienta alternativa primordial que suple a la educación virtual para los niños, jóvenes y adultos que no cuentan con conectividad en el desarrollo de su vida educativa. Además, se determinó que la educación y la comunicación mantienen una relación permanente y estrechamente ligada, pues la educación propone la incorporación de medios de comunicación con fines didácticos y prácticos al momento de la búsqueda de información, siendo parte fundamental del desarrollo y aprendizaje del ser humano.

De acuerdo con experiencias altamente productivas e interesantes ejecutadas en Ecuador y América Latina la educación a través de la radio ha generado resultados positivos en niños, jóvenes y adultos que cumplieron un proceso de alfabetización con el apoyo del Gobierno Nacional a través del Ministerio de Educación y las familias como actores fundamentales en el proceso educativo.

La radio sigue siendo para muchas zonas geográficas del país el principal contacto con el mundo exterior, elimina distancias y llega a lugares inaccesibles, amplía las oportunidades educativas para la población al reproducir o crear una extensión del aula.

Razón por lo que, es urgente e imperante crear espacios educativos, en donde exista la participación ciudadana, el estado y los medios, formado una fuerte trilogía, para así promover una educomunicación eficaz en lugares donde no existe conectividad. 


\section{REFERENCIAS}

Aguaded, J. I. (1995). La educación para la comunicación. La enseñanza de los medios en el contexto iberoamericano. Educación y Medios de Comunicación en el contexto iberoamericano, 19-48.

Arnheinm, R. (1979). Estética Radiofónica. Barcelona, España: Gustavo Gili

Arteaga, C. (2004). Un paso por la historia de la radio. Razón y Palabra.ERBOL.20

Bernal, C. A. (2006). Metodología de la Investigación (Segunda Edición ed.). México, México: Perason Educación

Guerrero, E. (12 de agosto de 2020). Corresponsabilidad de los medios radiales con la educación. Radio Latacunga. (V.
Peñaherrera, Entrevistador) Latacunga, Cotopaxi, Ecuador

Guaigua, S. (2 de agosto de 2020). Rol de los medios radiales. (V. Medina, Entrevistador) Latacunga, Cotopaxi, Ecuador

Hurtado, F. (julio de 2020). La educación en tiempos de pandemia. Revista Arbitrada del Centro de Investigación y estudios generales(44), 180

IRFEYAL. (2014). Cuarenta años de pasión por la educación. Quito, Ecuador: ediecuatorial

LOC, Ley Orgánica de Comunicación.(2013). Asamblea Nacional del Ecuador. Quito, Pichincha, Ecuador.

Villoro, L. (1989). Creer, saber, conocer. (S. XXI, Ed.) México, México 